\title{
The Causes, Diagnose and Intervention of Game Addiction
}

\author{
Mengqi Gong ${ }^{1, \mathrm{a}, \dagger}$, Yue $\mathrm{Hu}^{2, \mathrm{~b},{ }^{*}, \dagger}$, Dong Zhao ${ }^{3, \mathrm{c}, \dagger}$
}

\author{
${ }^{1}$ University of York, York, UK \\ ${ }^{2}$ Nanjing Normal University, Nanjing, $\mathrm{CHN}$ \\ ${ }^{3}$ Fuzhou University, Fuzhou, CHN \\ *Corresponding author.Email: ${ }^{*}$ mengqi.gong@foxmail.com, ${ }^{b} 192302006 @ n j n u . e d u . c n,{ }^{c 992568819 @ Q Q . c o m ~}$ \\ These authors contributed equally.
}

\begin{abstract}
Since the birth of video games, psychological research has focused on game addiction, and previous studies have obtained many valuable results. However, the researches on game addiction still have many limitations since they are lack integration. Therefore, this paper systematically introduces the research on game addiction: the causes, diagnostic criteria, and interventions of game addiction. We list the databases and screening criteria for searching the literature; 54 articles were included in this literature review. In the first part of this review, we discuss the causes of game addiction, including physiological factors, psychological factors, and external environments, and gave some suggestions about the causes of game addiction to further researchers. The second part compares three different diagnostic criteria such as Diagnostic and Statistical Manual of Mental Disorders (DSM-5) and International Classification of Diseases (ICD-11) from the American Psychiatric Association (APA) and World Health Organization (WHO), respectively, and the Clinical diagnostic criteria for Internet addiction from China. In the third part, we describe the two most commonly used interventions for game addiction: medication intervention and psychological intervention. In addition, we also summarize and analyze the effects of various therapies and their shortcomings.
\end{abstract}

Keywords: game addiction, causes of game addiction, DSM-5, ICD-11, intervention, CBT

\section{INTRODUCTION}

On August 3, 2021, the economic information daily published an article entitled " 'spiritual opium' has grown into an industry worth hundred billion ", in this article, the network game called new "drug" or "spiritual opium", which aroused a wide discussion among the public in China, and directly caused quite a stir in the capital market, total market value evaporated is close to 300 billion [1]. The latest event showed that gaming addiction had become a concern in China. According to ICD-11, people with gaming disorder would perform symptoms that make it difficult to control the amount of time playing video games and negatively impact their daily lives [2]. In China, the attention to game addiction is unprecedently high. Therefore, the impact of game addiction on people's physical and mental health and the inventions of video games has been a hot topic.

As the game-related industry has been widely concerned by all walks of life, the necessity of investigating game addiction has been revealed gradually. Researchers have coincidentally considered adolescents as the main object in this field, and most of the research methods were questionnaire surveys [3-7]. Most of the research topics about game addiction are its mechanism and causes, but these studies mainly focused on one specific field like biological mechanism or mental factors without a comprehensive view. And those studies mostly provided a western view of game addiction and noticed that only a few articles concerned the diagnosis and intervention in eastern culture. Therefore, this study will give an integrated review of game addiction on the causes of game addiction, the diagnosis criteria, and interventions. We also would consider the eastern culture.

In this article, the first part will discuss the antecedents of game addiction from both physiological and psychological perspectives. The second part will demonstrate the most known diagnostic criteria for 
game addiction worldwide and diagnostic criteria in China. In the third part, we summarized the intervention approaches commonly used to treat game addiction, including medication intervention in Eastern and Western countries. The psychological intervention mainly focuses on cognitive behavioral therapy (CBT), then demonstrates some potential problems. Lastly, we had a further discussion and concluded based on the three parts of this article.

\section{METHOD}

\subsection{Inclusion and Exclusion Criteria}

Inclusion criteria were that studies: 1) elaborate the diagnostic criteria of internet game addiction; 2) elaborate the causes of internet game addiction; 3) evaluate the effectiveness of interventions for internet game addiction.

Studies were excluded if they: 1) focused on prevention rather than treatment; 2) were not available in English. 3) come from SSCI and CSSCI journals. The database search was conducted on August 16, 2021.

\subsection{Search strategy}

We searched CNKI (China National Knowledge Infrastructure), Google Scholar, Web of Science using the following combination of search terms specified for "Title": ['internet game addiction' OR 'video game addiction' OR 'online game addiction'] AND ['diagnostic criteria' OR 'cause' OR 'treatment' OR 'intervention'] AND ['DSM-5' OR 'ICD-11'].

\subsection{Results of screening}

This study has obtained 58 articles about internet game addiction with its diagnostic criteria, causes, and interventions. The time of these articles was from 1996 to 2020 .

\section{CAUSES OF GAME ADDICTION}

Game addiction is one of the most common forms of internet addiction and contains the concept of non-drug addiction [8]. Non-drug addictions have some similar mechanisms and causes to drug addictions. Therefore, some research results about drug addiction's causes and mechanisms can also reveal the causes of game addiction. Studies on the form of game addiction provide references for the research of other addictions as well. We can conclude that game addiction causes include physiological factors, personality characteristics, and influences of external environments.

\subsection{Physiological factors}

Psychologists and psychiatrists have conducted many researches to examine the brain physiological mechanism of addiction. They found that substance addiction and non-substance addiction have a common biological basis [9]. And, game addiction is accompanied by a certain degree of damage to brain structure and function [10]. All kinds of addiction involve four brain cognitive functional circuits: reward, cognitive control, motivation and memory-learning [11]. Damage and abnormal neural activity in the relevant circuits of the brain area will lead to the symptoms of addiction.

\subsubsection{Reward circuit}

Reward Deficiency Syndrome (RDS) theory considered that the damage to the striatum of the limbic system could lead to game addiction [12]. The striatum is the center of the human brain's reward and is very sensitive to reward increase. RDS theory deems that the decrease of striatum activity leads to decreased dopamine secretion when the human brain faces the reward of the same value. Therefore, patients need out more game activities to compensate for the same degree of dopamine secretion. A lack of one gene may cause the striatum to lack enough dopamine receptors to produce a response to reward [12].

\subsubsection{Anterior cingulate gyrus, thalamus and occipital gyrus}

Game addiction is related to the abnormality of the striatum and reward circuit and related to the injury of anterior cingulate gyrus, thalamus, and occipital gyrus [10]. A comparative study on the brain structure of online game addicts, professional game players and normal control group showed that the gray matter volume of the left anterior cingulate gyrus is decreased, and the gray matter of volume of inferior temporal cortices and occipital cortices also have significant differences [13]. These abnormal brain structures may be related to the illness of game addicts.

\subsubsection{Abnormal of the prefrontal cortex}

The abnormal prefrontal cortex is also related to game addiction [11, 14]. The prefrontal cortex is the center of human cognitive function control. If this part of the cortex has an injury, it will lead to the abnormality of the cognitive control circuit in patients with game addiction. Game addiction involves the dysfunction of many parts of brain circuits, and there are interactions between these mental disorders. Psychologists and psychiatrists are still doing further study on the neural mechanism of game addiction.

\subsection{Heredity factors}

Psychologists and biologists also try to explore 
whether genetic factors impact addiction, and the results showed that it is. Black's investigation [15] showed that shopping addiction is hereditary. Eisen's research [16] found a higher incidence rate of pathological gambling among people whose first-degree relatives also have pathological gambling disorders. Beaver [17] indicated that the heredity of non-drug addiction has gender differences which means it can explain males' addiction but cannot explain female addiction. In the future, researchers will explore the physiological mechanism of addiction from a genetic perspective [8].

At present, most studies on this field only focus on individual brain regions. However, the functions of individual brain regions are not isolated. In the future, psychologists can further explore the interaction between the functions of various brain regions related to game addiction and further reveal the psychological factors of game addiction.

\subsection{Mental factors}

According to previous studies, the psychological factors of game addiction mainly include personality traits and comorbidity of other mental diseases.

\subsubsection{Personality traits}

Personality traits can predict which kind of games that people will addict [18]. A survey of game addiction among Junior students in Korea found that game types have unique psychological tendencies than other mental diseases, affecting their game choice and internet use [18]. Kim's research showed that aggressive and narcissistic personality characteristics are positively correlated with online game addiction, while self-control is negatively correlated with game addiction. Susana's investment [19] showed that people with low self-esteem might be play games to access more self-esteem. Psychological states like loneliness and social anxiety also greatly influence game addiction[18] (Kim et al., 2008).

\subsubsection{Comorbidity of other mental disorders}

Many studies have shown a co-occurrence relationship between game addiction and poor mental health [20]. Video game addiction is related to increased stress, anxiety and depression [21]. Loton found [13] the greatest relationship between video game addiction and anxiety, followed by stress and depression, which confirms the correlation between game addiction and poor mental health. Brunborg's research [20] shows that video game addiction is associated with higher levels of depression, poor academic performance and more behavioral problems.

Game addiction is likely to be the manifestation of other psychological problems. Finding the relationship between these psychological factors and game addiction plays an important role in the treatment. According to these psychological characteristics, potential game addiction patients can be screened and diagnosed clinically, and intervention can be carried out according to the corresponding psychological problems.

\subsubsection{Influences of external environments}

By searching articles in the Web of Science and Chinese academic paper platform, we found that the objects of game addiction mainly are adolescents, which showed that adolescents are a high incidence group of game addiction. Teenagers' psychological function and nervous system are immature. The fascinating visual form, attractive plot and joyful social functions in the online game are very attractive to them [22]. The stimulation of the external environment is likely to lead to the pathological attack of game addiction. Among many external stimuli, Parenting styles and the use of media have a strong influence.

\subsubsection{Parenting styles}

Wang's study [23] on Chinese high school students' computer game addiction shows that children's game addiction tendency is more serious under the autocratic parental rearing style. Shek's study [24] found that the quality of parental psychological control and personal relationship predicted adolescent addiction behavior, in which mothers could predict the changes of adolescent addiction and crime. These findings suggested that parental autocracy may lead minors to get compensation from computer games, resulting in an addiction to games. In addition, low parental support and parents' addiction to games will also increase the probability of minors' game addiction.

\subsubsection{Use of media}

Media also influence the process of game addiction among teenagers group. Sümen's survey [25] found that Internet addiction will negatively impact teenagers' psychological state. Rehbein's research [26] believed that for children before the age of 10 , parents' active control and reducing access to game-related media could help prevent children from suffering from game addiction in adolescence, but for children over the age of 10, the effect of using this method is extremely limited.

In addition to family rearing styles and media, other external factors impact game addiction, such as school factors. Some studies have found that students with school phobia are more likely to indulge in online games [26], and school connectedness can help reduce game addiction [27]. Further research should reveal more external factors affecting minors' game addiction so that adolescents' guardians can protect them from 
these external stimuli and prevent them from becoming game addiction patients.

\section{DIAGNOSTIC CRITERIA FOR GAME ADDICTION}

Researchers and healthcare professionals still wonder whether gaming addiction should be regarded as a kind of disease [28]. However, gaming addiction has been included in the American Psychiatric Association's DSM-5, which recognizes online gaming disorders and caffeine use disorders and other conditions in the section recommending further research conditions [28, 29]. Similarly, the WHO defined gaming disorder as a new disorder in the ICD-11 [30]. In addition to these two common diagnostic criteria in Western countries, Chinese scholars published Clinical diagnostic criteria for Internet addiction [31]. Thus, we will look at how gaming addiction is diagnosed in the three diagnostic criteria mentioned above and compare.

\subsection{Diagnostic and Statistical Manual of Mental Disorders (DSM-5)}

The DSM-5 stated that "game disorder" is only diagnosed when gaming causes "significant impairment or distress" to several aspects of a person's life and when at least five of the following nine symptoms are experienced within a year [28, 29]. Namely, 1) Engrossed in the game; 2) Withdrawal symptoms similar to sadness, anxiety, irritability when the game is canceled or when the person cannot play; 3) Show tolerance and an increasing desire to play games that cannot be satisfied without spending more time playing; 4) Unable to play less and quit the game successfully; 5) Loss of interest in previously enjoyed activities; 6) Continuing to the game despite problems; 7) Lying to family or others about playtime; 8) Using games to relieve negative emotions, such as guilt or despair; 9) Nearly losing or facing the risk of losing a job or a relationship due to excessive gaming [28].

In 2014, the international consensus for assessing internet gaming disorder described the nine symptoms proposed by the DSM-5 in more detail and developed key elements of epidemiological research on the subject. This movement has led to a convergence of research in defining play using the nine symptoms proposed by the DSM-5 [32]. In the nearly 20 years since IGD was included in the DSM-5, this criterion has gradually become the most accepted in this area, and there is a growing number of studies in this area that aim to provide DSM-5 prevalence estimates for IGD and to evaluate different tools for assessing the disorder [33].

\subsection{The International Classification of Diseases (ICD-11)}

The 11th Revision of the International Classification of Diseases (ICD-11) defines 'gaming disorder' as a pattern of gaming behavior ('digital gaming' or 'video gaming'), and the symptoms are described in three ways only: impaired control overplay, increased prioritization of play, and continued or escalating dependence on the play despite negative consequences $[30,33]$.

In comparison, both the DSM-5 and ICD-11 include clinically significant impairment or distress in the clinical diagnosis, and both review the subject's experience in the past 12 months, but the symptoms described in ICD-11 are much vaguer. A study used a sample of 7,022 young adults in Mexico to examine the diagnostic effects of DSM-5 and ICD-11. As a result, the number of subjects diagnosed with game disorder according to DSM-5 criteria was almost twice as high as the ICD-11 criteria. When researchers compared the two groups of addicts' cases detected by DSM-5, one group of them not found by ICD-11 and both DSM-5 and ICD-11 identified another group, the result did not found any significant differences. There appeared to be little reason to intervene in one group over the other [33]. Therefore, using only ICD-11 as a diagnostic criterion may lead people with a similar behavioral disorder that could not be intervened in time, so that DSM-5 may be a more appropriate diagnostic criterion for gaming disorder.

\subsection{Chinese Clinical diagnostic criteria for Internet addiction}

Besides DSM-5 and ICD-11, there are diagnostic criteria for internet addiction in China: clinical diagnostic criteria. Although the criterion is not focused on gaming addiction, $87 \%$ of the cases studied in this criterion are from gaming addiction [34]. ISnternet addiction is a mental, behavioral disorder caused by repeated and excessive Internet use, including gaming addiction [34]. The criteria for Internet addiction are an average of six or more hours of continuous Internet use per day and having met the following criteria for symptoms for three months or more [31] : Firstly, the addicts should performance a strong craving or urge to use the Internet; Secondly, there are some withdrawal reactions such as peripheral discomfort, irritability, irritability, inattention, sleep disturbance when reducing or stopping Internet use, which can be alleviated by using other similar electronic media such as television, handheld game consoles, etc. The third point included five sub-points, and at least one of the following has met this point would be satisfied: 1) Increasing time and commitment to Internet users to achieve satisfaction; 2) Difficulty in controlling the beginning, end and duration of Internet use, after repeated unsuccessful attempts; 3) 
Persistent use of the Internet despite its obvious harmful consequences and difficulty in stopping even when the harmful effects of Internet use are known; 4)Reduction or abandonment of other interests, recreational or social activities as a result of Internet use; 5) Using the Internet as an escape from problems or as a way to relieve bad feelings.

The official diagnostic criteria for gaming addiction in China are limited until now. And even though gaming addictions developed the majority cases of the study, other behavioral disorders such as social networking addiction are still needed to be considered. Comparing with DSM-5 and ICD-11 criteria, the Chinese Clinical diagnostic criteria for Internet addiction is more stringent. To be more specific, the Chinese criteria only observe participant's behaviors over the past three months, but both DSM-5 and ICD-11 retrospect to 12 months ago. Moreover, a previous study showed that approximately two-thirds of the participants reported increased Internet use during the pandemic of coronavirus disease in 2019, which is approximately three months, with more than $10 \%$ of participants accessing the Internet for over 8 hours per day. Also, many other activities have been replaced by Internet using [35]. As a result, those participants would be diagnosed as addicts by mistake using the Clinical diagnostic criteria for Internet addiction. However, they might move their working procedure and social life online, which indicated that these criteria might not be appropriate in this circumstance.

Many clinical diagnoses of game addiction still did not use the scale specific to games based on DSM-5 or ICD-11, but rather internet addiction scales such as Young's Internet Addiction Test [36] and Chen's Internet Addiction Scale [37], with only a few studies using scales that meet the DSM-5 criteria, for instance, the Clinical Video Game Addiction Test (C-VAT 2.0) which contains three questions about gaming, and 11 dichotomous questions about game disorder behaviors based on participant's experience during the previous years on account of the nine DSM-5 criteria for Internet Game Disorder. To sum up, the common standard and shared criteria for measuring Game Disorder have not yet been determined, and no consensus has been reached on this area [38].

Therefore, according to the information demonstrated above, several concerns need to be brought up: First of all, comparing with the other two criteria, DSM-5 could be regarded as the most accepted one, the ICD-11 might fail to detect some addicts requiring intervention. In contrast, the Chinese criteria might treat some normal behaviors as addictions. Secondly, the diagnoses of game addiction in China are still not detailed enough. Specifically, the case study needs to be up to date and focus on gaming addiction only rather than the broader concept of internet addiction. Similarly, most of the world's clinical diagnoses of gaming addiction did not use scales based on the DSM-5 or ICD-11 criteria, so the next step should be to promote the usage of diagnostic criteria for gaming addiction.

\section{INTERVENTION}

The earliest research about game addiction originated in the late 1990s [39]. Since then, how to intervene the game addiction effectively has been a hot topic of social issues. Through more than 20 years of studies, researchers have intervened with many people with game addiction, which was remarkable [40, 41, 42]. Our review summarized two popular intervention methods: psychological intervention and medication intervention [43].

\subsection{Medication Intervention}

In recent years, medication intervention has been used in other behavioral or substance addictions, like gambling addiction and drug addiction, and plays an important role in game addiction intervention. Most studies using drugs for game addition intervention were conducted in western countries and South Korea [44].

The main medication currently used to treat game addition are antidepressants, opioid receptor antagonists, mood stabilizers and antipsychotics. Sattar and Ramaswamy firstly used antidepressants to treat game addiction [45]. In their study, a man with severe depression and game addiction received treatment with Escitalopram at the dose of $30 \mathrm{mg} / \mathrm{d}$ for 3 months. The result showed that these drugs not only improved his depression but also reduced his desire for online games. Bupropion was also used in the treatment of patients with substance dependence. In another study, researchers used Bupropion to alleviate the desire for the Internet of the game addiction participants. This study contained a 12-week random double-blind clinical treatment, including 8 weeks of active treatment and 4 weeks of Follow-up test. The results showed that the participants' desire for online games and depression reduced [46].

However, the studies on the use of drugs to treat game addiction were limited worldwide, and there was a lack of research on medication intervention in-game addiction in Chinese culture [44]. In addition, the drug intervention mainly focused on the situations of the game addiction with depression and anxiety. However, people who only have game addiction may be less willing to take medication to treat their addiction. Therefore, researchers often did not use the medication intervention alone in some studies but use it with psychological intervention [47]. The combination of interventions can ensure the stability and balance of the addict's physiological system and help the patient 
eliminate false cognitions and establish appropriate and adaptive cognitive ways [48].

\subsection{Psychological Intervention}

Psychological intervention methods include cognitive therapy, behavior therapy, cognitive behavioral therapy, family therapy, group-based treatment, etc. Cognitive-behavioral therapy (CBT) is the most widely used psychological intervention method to game addiction. Through personal time management, self-motivation and support systems, CBT helps participants establish effective processing strategies, change addictive behaviors and achieve ideal goals.

\subsubsection{Cognitive Behavioral Therapy-Internet Addiction (CBT-IA)}

Young believes that internet game addiction is very different from other substance addictions, and there were three characteristics of the cause of internet addiction: anonymity, convenience, and escape [49]. So, it is very difficult to adopt the traditional intervention therapy for internet game addiction. Therefore, Young designed the CBT-IA (Cognitive Behavioral Therapy-Internet Addiction), cognitive behavioral therapy for internet game addiction [50]. The therapy consists of three phases: behavior modification, cognitive restructuring and harm reduction therapy.

The first stage is behavior modification, which reduces or controls the internet gaming behavior by forcing the addict to get rid of the emotional state of control and trance. The second stage, cognitive reconstruction, makes addicts understand that these thoughts like "they cannot get love and value in real life" are negative perceptions and playing online games can only get temporary substitute satisfaction. In addition, the therapist should guide them to eliminate resistances gradually. The third stage is harm reduction therapy. At this stage, therapists should help solve other problems of addicts, such as depression, anxiety and low self-esteem. Young used CBT-IA to measure its treatment outcomes with 128 internet addicts. The results showed that more than $95 \%$ of participants could control their symptoms by the end of 12 weeks, and $78 \%$ of them could keep them 6 months after treatment [51].

\subsubsection{Mindfulness-oriented Recovery Enhancement (MORE)}

Another type of CBT therapy for internet game addiction is mindfulness-oriented recovery enhancement (MORE). MORE is a new method to treat internet game addiction. It combines mindfulness training, "Third Wave" cognitive-behavioral therapy, and positive psychological principles that specifically address addictions and co-occurring mental distress $[52,53,54]$. $\mathrm{Li}$ et al. used MORE to treat internet game addiction. A total of 30 internet game addicts were randomly assigned to either the intervention group or the control group to receive MORE or support group treatment for 8 weeks. The results showed that the intervention group had significantly fewer IGD criteria, fewer video game cravings, and maladaptive cognitions associated with gaming compared with the control group. Furthermore, the treatment effect remained unchanged during the 3-month-follow-up test [55].

However, some problems still exist in most studies with psychological intervention. Firstly, whether in Eastern or Western countries, the control group is less used in research design to analyze and compare the effects of psychological intervention. So, it is difficult for researchers to rule out the influence of Rosenthal's effect on the experiment results. In addition, most studies report the baseline data, sample size, and post-test data but do not report the specific intervention process, which makes it difficult to compare the intervention effects of the same therapies. Finally, the sample size in most studies is small and lacks follow-up testing, which might limit our abilities to detect true differences if they were indeed present $[56,57]$.

\section{DISCUSSION}

This paper discussed game addiction in detail from three perspectives: causes, diagnostic criteria and interventions.

As for the causes of game addiction's physiological, psychological, and external factors, respectively $[8,13$, 18], with the deepening of research, researchers found that the causes of game addiction were very complex. The game addiction depends on the interaction between physiological, psychological, and external factors[14]. However, researchers have not figured out what the interaction is and how the interaction influences the cause of game addiction[14]. In the future, there are two aspects researchers should do to go deeper in this field: First, they should study the mechanism of a single factor and its influence on game addiction more specifically; Second, researching how the interaction among physiological, psychological and external factors affect the cause of game addiction.

When it comes to the diagnosis of game addiction, the most mainstream diagnostic standard in the world is DSM-5. In comparison, the diagnostic standard of ICD-11 is very strict, leading to some potential game addicts not being correctly diagnosed [29, 30, 33]. However, the clinical diagnostic criteria for Internet addiction standard in China is relatively loose, which may diagnose game addiction if the patient does not reach the degree of game addiction. With the development of the Internet and games, the definition of 
game addiction might vary with different circumstances, to be more specific, the prevalence of electronic devices and whether they are in the pandemic, and the criteria for diagnosing game addiction should not be one-size-fits-all but up to date [35]. At the same time, there are other auxiliary diagnostic criteria. For example, in the clinic, psychological counselors and psychiatrists can also make the correct diagnosis with the help of some physiological indicators, patients' self-assessment scales, interviews and self-statements. However, the diagnosis of online game addiction worldwide should reach a basic consensus and develop further on this basis, which may be the next step for psychologists and scholars [37].

The treatments of game addiction are mainly psychological intervention, medication intervention [43]. On the one hand, medication intervention is usually used when game addiction is comorbid with other mental diseases like depression and anxiety. In addition, compared with Western countries, there were relatively few studies on the use of medication to treat gaming addiction in China [56, 57], so it is very important to verify the effectiveness of medication intervention in the Chinese environment. On the other hand, at present, the major psychological intervention is cognitive-behavioral therapy (CBT), and its effect on gaming addiction has been proved in the context of eastern and western cultures [43]. Moreover, more therapy combined with mindfulness is a new form of CBT therapy which has an excellent effect, but the duration of intervention effect was still needed to be further explored $[52,53,54]$. In the future, clinical psychologists can also integrate drug and non-drug treatments to balance treatment effectiveness, duration, and minimum damage to patients' bodies [57].

\section{CONCLUSION}

In general, this review has combed the current game addiction research among causes, diagnosis, and intervention. Further research can focus on the interaction of the factors that influence game addiction; improve the diagnosis criteria for adolescents; make the criteria suit people from different cultures and design intervention with a more lasting effect.

\section{REFERENCES}

[1] Wu, L. (2021). An article triggered an avalanche, the game was criticized as "opium of the mind"! What does the sudden change of tongue mean? STCN. Retrieved

from. https://news.stcn.com/sd/202108/t20210804 35066 52.html.

[2] World Health Organization. (2018). Addictive behaviours: Gaming disorder. Retrieved August 19 2021,

from https://www.who.int/news-room/q-a-detail/addictiv e-behaviours-gaming-disorder .

[3] Essau, C. A., \& Delfabbro, P. (Eds.). (2008). Adolescent addiction: Epidemiology, assessment, and treatment.

[4] Lemmens, J. S., Valkenburg, P. M., \& Peter, J. (2009). Development and validation of a game addiction scale for adolescents. Media psychology, 12(1), 77-95.

[5] Griffiths, M. D. (2010). Adolescent video game addiction: issues for the classroom. Education Today, 60(4), 32-34.

[6] Van Rooij, A. J., Schoenmakers, T. M., Vermulst, A. A., Van Den Eijnden, R. J., \& Van De Mheen, D. (2011). Online video game addiction: identification of addicted adolescent gamers. addiction, 106(1), 205-212.

[7] Aziz, N., Nordin, M. J., Abdulkadir, S. J., \& Salih, M. M. M. (2021). Digital Addiction: Systematic Review of Computer Game Addiction Impact on Adolescent Physical Health. Electronics, 10(9), 996.

[8] Li, M.-J., Chen, J., \& Li, X.-Y. (2013). Genetic and Neurobiological Mechanisms of Non-drug Addictions. Advances in Psychological Science, 20(10), 1623-1632.

[9] Doug Hyun Han, Y. S. K., Yong Sik Lee, Kyung Joon Min, and Perry F. Renshaw, . (2010). Changes in Cue-Induced, Prefrontal Cortex Activity.

[10] Han, D. H., Lyoo, I. K., \& Renshaw, P. F. (2012, Apr). Differential regional gray matter volumes in patients with online game addiction and professional gamers. J Psychiatr Res, 46(4), 507-515

[11] He, J., Nie, Y., Zhou, Z., \& Chai, Y. (2017). Are both neural mechanisms of Internet gaming and heroin addicts the same? Research evidence based on MRI. Advances in Psychological Science, 25(8).

[12] Kenneth Blum, E., Gardner, Marlene Oscar-Berman and Mark Gold. (2012). "Liking" and "wangting" Linked to Reward Deficiency Syndrome(RDS):Hypothesizing differential Responsivity in Brain Reward Circutry. Current Pharmaceutical Design, 18(1), 113-118.

[13] Loton, D., Borkoles, E., Lubman, D., \& Polman, R. (2015). Video Game Addiction, Engagement and Symptoms of Stress, Depression and Anxiety: The Mediating Role of Coping. International Journal of Mental Health and Addiction, 14(4), 565-578.

[14] HE Jin- bo, G. Y.-y., XIANG Yuan- ming. (2008). 
Forming Mechanism of Adolescents'Internet- game Addiction. Chinese Journal of Clinical Psychology, $16(1), 46-48$

[15] Donald W.Black, M. D., Susan Repertinger, M.D., Gray R. Gaffney, M.D., and Janelle Gabel,R.N. (1998). Family History and Psychiatric Comorbidity in Persons With Compulsive Buying: Preliminary Findings. Am J Psychiatry, 155(7), 960-963.

[16] Seth A. Eisen, N. 1., Michael J. Lyons, Jeffery F. Scherrer, Kristin Griffith, William R. True, Jack Goldberg \& MIng T.Tsuang. (1998). Familial influences on gambling behavior:an analysis of 3359 twin pairs. Addiction, 1375-1384.

[17] Beaver, K. M., Hoffman, T., Shields, R. T., Vaughn, M. G., DeLisi, M., \& Wright, J. P. (2010, Mar). Gender differences in genetic and environmental influences on gambling: results from a sample of twins from the National Longitudinal Study of Adolescent Health. Addiction, 105(3), 536-542.

[18] Kim, E. J., Namkoong, K., Ku, T., \& Kim, S. J. (2008, Apr). The relationship between online game addiction and aggression, self-control and narcissistic personality traits. Eur Psychiatry, 23(3), 212-218.

[19] Jimenez-Murcia, S., Fernandez-Aranda, F., Granero, R., Choliz, M., La Verde, M., Aguglia, E., Signorelli, M. S., Sa, G. M., Aymami, N., Gomez-Pena, M., del Pino-Gutierrez, A., Moragas, L., Fagundo, A. B., Sauchelli, S., Fernandez-Formoso, J. A., \& Menchon, J. M. (2014). Video game addiction in gambling disorder: clinical, psychopathological, and personality correlates. Biomed Res Int, 2014, 315062.

[20] Brunborg, G. S., Mentzoni, R. A., \& Froyland, L. R. (2014, Mar). Is video gaming, or video game addiction, associated with depression, academic achievement, heavy episodic drinking, or conduct problems? J Behav Addict, 3(1), 27-32.

[21] Mentzoni, R. A., Brunborg, G. S., Molde, H., Myrseth, H., Skouveroe, K. J., Hetland, J., \& Pallesen, S. (2011, Oct). Problematic video game use: estimated prevalence and associations with mental and physical health. Cyberpsychol Behav Soc Netw, 14(10), 591-596.

[22] Floros, G., Siomos, K., Stogiannidou, A., Giouzepas, I., \& Garyfallos, G. (2014, Oct). The relationship between personality, defense styles, internet addiction disorder, and psychopathology in college students. Cyberpsychol Behav Soc Netw, $17(10), 672-676$.
[23] WANG Pan, G. Y.-q., LI Min. (2006). Resear ch on Relationship Between Tendency to Computer Game addiction and Par ental Rearing Styles in Senior High School Students. Chinese Journal of Clinical Psychology, 14(5),460-462.

[24] Shek, D. T. L., \& Law, M. Y. M. (2014). Parental Behavioral Control, Parental Psychological Control and Parent-Child Relational Qualities: Relationships to Chinese Adolescent Risk Behavior. In Chinese Adolescents in Hong Kong (pp. 51-69).

[25] Sümen, A., \& Evgin, D. (2021). Social Media Addiction in High School Students: A Cross-Sectional Study Examining Its Relationship with Sleep Quality and Psychological Problems. Child Indicators Research.

[26] Rehbein, F., \& Baier, D. (2013). Family-, Media-, and School-Related Risk Factors of Video Game Addiction. Journal of Media Psychology, 25(3), 118-128.

[27] Zhu, J., Zhang, W., Yu, C., \& Bao, Z. (2015). Early adolescent Internet game addiction in context: How parents, school, and peers impact youth. Computers in Human Behavior, 50, 159-168.

[28] Parekh, R. (2018). Internet Gaming. Retrieved August 17 2021, from https://www.psychiatry.org/patients-families/intern et-gaming .

[29] American Psychiatric Association. (2013). Diagnostic and Statistical Manual of Mental Disorders (DSM-5®) American Psychiatric Publishing.

[30] World Health Organization. (2018). The ICD-11 Classification of Mental and Behavioral Disorders: Diagnostic Criteria for Research.

[31] Tao, R., et al. (2008). Clinical diagnostic criteria for Internet addiction (1st ed.). Beijing: General Hospital of Beijing Military Region.

[32] Petry, N. M., Rehbein, F., Gentile, D. A., Lemmens, J. S., Rumpf, H. J., Mößle, T., ... \& O'Brien, C. P. (2014). An international consensus for assessing internet gaming disorder using the new DSM-5 approach. Addiction, 109(9), 1399-1406.

[33] Borges, G., Orozco, R., Benjet, C., Mart' inez, K. I. M. I., Contreras, E. V., P' erez, A. L. J. E., ... \& Ayuso-Mateos, J. E. L. (2020). (Internet) Gaming Disorder in DSM-5 and ICD-11: A Case of the Glass Half Empty or Half Full:(Internet) Le trouble du jeu dans le DSM-5 et la CIM-11: Un cas de verre à moitié vide et à moitié plein. The Canadian Journal of Psychiatry, 66(5), 477-484. 
[34] Liu, X., Wang, J., \& Zhuang, H. (2008). "Clinical diagnostic criteria for Internet Addiction" through expert proof. Xinhua News.

[35] Jovic, J., Pantovic-Stefanovic, M., Mitkovic-Voncina, M., Dunjic-Kostic, B., Mihajlovic, G., \& Milovanovic, S. et al. (2020). Internet use during coronavirus disease of 2019 pandemic: Psychiatric history and sociodemographics as predictors. Indian Journal Of Psychiatry, 62(9), 383.

[36] Young, K. S. (1996). Psychology of computer use: XL. Addictive use of the Internet: a case that breaks the stereotype. Psychological reports, 79(3), 899-902.

[37] Chen, S. H., Weng, L. J., Su, Y. J., Wu, H. M., \& Yang, P. F. (2003). Development of a Chinese Internet addiction scale and its psychometric study. Chinese Journal of Psychology.

[38] Costa, S., \& Kuss, D. J. (2019). Current diagnostic procedures and interventions for Gaming Disorders: A Systematic Review. Frontiers in psychology, 10, 578.

[39]Young, K. S. (1996). Internet Addiction : The emergence of a new clinical disorder. the 104th annual meeting of the American Psychological Association in Toronto, Canada. August 20,1996.

[40] Sujin, B. , Hong, J. S. , Mi, K. S. , \& Han, D. H. . (2018). Bupropion shows different effects on brain functional connectivity in patients with internet-based gambling disorder and internet gaming disorder. Frontiers in Psychiatry, 9, 130-

[41] Yao, Y. W., Chen, P. R., Chiang-shan, R. L., Hare, T. A., Li, S., Zhang, J. T., ... \& Fang, X. Y. (2017). Combined reality therapy and mindfulness meditation decrease intertemporal decisional impulsivity in young adults with Internet gaming disorder. Computers in Human Behavior, 68, 210-216.

[42] Kim, P. W. , Kim, S. Y., Shim, M. , Im, C. H. , \& Shon, Y. M. . (2013). The influence of an educational course on language expression and treatment of gaming addiction for massive multiplayer online role-playing game (mmorpg) players. Computers \& Education, 63(apr.), 208-217.

[43] Kristyn Zajac, Meredith K. Ginley \& Rocio Chang. (2019). Treatments of internet gaming disorder: a systematic review of the evidence. Expert Review of Neurotherapeutics, 20(1).

[44] Camardese, G. , Risio, L. D. , MD Nicola, Pizi, G. , \& Janiri, L. . (2012). A role for pharmacotherapy in the treatment of "internet addiction". Clinical Neuropharmacology, 35(6), 283-289.

[45] Sattar, P. , \& Ramaswamy, S. . (2005). Internet gaming addiction. Canadian journal of psychiatry. Revue canadienne de psychiatrie, 49(12), 869-870.

[46] Doug, Hyun, Han, Jun, Won, \& Hwang, et al. (2010). Bupropion sustained release treatment decreases craving for video games and cue-induced brain activity in patients with internet video game addiction. Experimental \& Clinical Psychopharmacology.

[47] Przepiorka, A. M. , Blachnio, A. , Miziak, B. , \& Czuczwar, S. J. . (2014). Clinical approaches to treatment of internet addiction. Pharmacological Reports.

[48] Alexander Winkler, Beate Dörsing, Winfried Rief, Yuhui Shen, Julia A. Glombiewski (2013). Treatment of internet addiction: a meta-analysis. Clinical Psychology Review, 33(2), 317-329.

[49] Young, K. S. (1999). Internet addiction: symptoms, evaluation, and treatment. innovations in clinical practice a source book.

[50] Young, K. S.(2011). Cbt-ia: the first treatment model for internet addiction. Journal of Cognitive Psychotherapy, 25(4).

[51]Young, K. S. (2013). Treatment outcomes using cbt-ia with internet-addicted patients. Journal of Behavioral Addictions, 2(4).

[52] Garland, E. L., Gaylord, S. A., Boettiger, C. A., \& Howard, M. O. (2010). Mindfulness training modifies cognitive, affective, and physiological mechanisms implicated in alcohol dependence: Results of a randomized controlled pilot trial. Journal of Psychoactive Drug.

[53] Garland, E. L., Froeliger, B., \& Howard, M. O. (2014b). Mindfulness training targets neurocognitive mechanisms of addiction at the attentionappraisal-emotion interface. Frontiers in Psychiatry, 4, 173.

[54] Garland, E. L. (2016). Restructuring reward processing with mindfulnessoriented recovery enhancement: Novel therapeutic mechanisms to re-mediate hedonic dysregulation in addiction, stress, and pain. Annals of the New York Academy of Sciences, 1373, 25-37.

[55] Li, H. , \& Wang, S. . (2013). The role of cognitive distortion in online game addiction among chinese adolescents. Children \& Youth Services Review, 35(9), 1468-1475. 
[56] Liu, C., Liao, M., \& Smith, D. C. (2011). An empirical review of internet addiction outcome studies in china. Research on Social Work Practice, 22(3), 282-292.
[57] Zajac, K., Ginley, M. K., Chang, R., \& Petry, N. M. . (2017). Treatments for internet gaming disorder and internet addiction: a systematic review. Psychol Addict Behav, 31(8), 979-994. 